\title{
Transition from Lamb waves to longitudinal waves in plates
}

\author{
D W Greve ${ }^{1,4}$, P. Zheng, ${ }^{2}$ and I J Oppenheim ${ }^{3}$ \\ ${ }^{1}$ Department of Electrical and Computer Engineering, Carnegie Mellon University, \\ Pittsburgh, PA 15213 USA; Email: dg07@andrew.cmu.edu \\ ${ }^{2}$ Department of Physics, Carnegie Mellon University, Pittsburgh, PA 15213 USA; \\ Email: pengzhen@andrew.cmu.edu \\ ${ }^{3}$ Department of Civil and Environmental Engineering, Carnegie Mellon University, \\ Pittsburgh, PA 15213 USA; Email: ijo@cmu.edu
}

\begin{abstract}
We use finite element simulations and experiments to examine the excitation of waves in a plate by normal forces applied to an edge. At low values of the frequency-plate thickness product, $f \cdot d$, multiple Lamb wave modes are excited with different group velocities, but increasing $f \cdot d$ leads to the formation of a train of well-defined nearly-longitudinal pulses. We investigate the transition between these two wave types, and for the train of nearlylongitudinal pulses we determine the rate at which energy is transferred from the leading pulse to the trailing pulses. Finally, we examine the potential of these longitudinal waves for flaw detection. Experiments and simulations show that the longitudinal pulse train is reflected or transmitted from a slot without significant mode conversion.
\end{abstract}

\section{Introduction}

Steel highway bridges are typically constructed using plate girders or box girders. The initiation and growth of fatigue cracks is a primary concern during the life of the bridge, and is the subject of biennial inspections. A concern of highest priority is crack growth in flanges under tension, because a crack could reach fracture-critical length. Although visual inspection methods are widely used at present these are labor-intensive and are unreliable for the detection of small cracks [1]. Consequently improved methods for crack detection in critical areas would improve civil infrastructure safety. Moreover, some girders contain details that partly or totally obscure visual inspection, in which case ultrasonic methods for crack detection would provide the most reliable indication of structural safety. The flange edge is generally accessible, and therefore we investigate ultrasonic waves excited by edge loading of a plate.

Substantial research effort has been directed at the detection of defects in plate-like structures using ultrasonic Lamb waves, motivated by the fact that such guided waves propagate with low attenuation for considerable distances in plates. However, we note two major reasons to consider longitudinal waves (bulk waves, p-waves) as a alternative and possibly superior choice. For bulk waves a range of at least two meters, which is ample for most inspection requirements, is easily attained for bulk waves when excited either by commercial ultrasonic field equipment or by digital data acquisition systems. More significantly, flaw detection using Lamb waves remains a substantial challenge because reflections from flaws in the size range of practical interest are not easily distinguished from other waveforms. In this paper we investigate the transition from Lamb wave response to longitudinal wave

\footnotetext{
${ }^{4}$ To whom any correspondence should be addressed.
} 
response, and show that the nearly-longitudinal waves retain their well-defined characteristics after reflecting from flaws.

The challenge to using Lamb waves for flaw detection in steel plate girders originates in the fact that there exist multiple Lamb wave modes when the frequency-thickness product $f \cdot d$ exceeds, say, 3 $\mathrm{MHz} \cdot \mathrm{mm}$. As a result, many researchers recommend operating at low $f \cdot d$ products so that only a limited number of modes are excited [2,3,4], and other work addresses the development of transducers that selectively emit particular modes [5,6,7]. However, limiting transducer operation to low frequencies is generally not a satisfactory solution because scattering becomes weaker and the resolution is seriously degraded when the defect is small compared to the wavelength $[8,9,10,11,12]$.

In this paper we consider the creation of waves in plates by edge excitation at $f \cdot d$ products that correspond to multiple Lamb wave modes. For relatively low values of $f \cdot d$ we observe that Lamb wave modes are generated, at large $f \cdot d$ we observe longitudinal waves somewhat influenced by reflections from the surface, and for a range of intermediate $f \cdot d$ we observe a pulse train of nearly longitudinal waves. These regularly spaced "trailing pulses" have been discussed in the literature, but we are not aware of any investigations of the transition from Lamb wave generation at low $f \cdot d$ to the formation of nearly longitudinal waves and trailing pulses at higher $f \cdot d$. In addition, we examine the transport of energy from leading to trailing pulses. Finally, we consider the interaction of these trailing pulses with cracks. The results suggest that these nearly longitudinal waves are an attractive option for flaw detection.

\section{Excitation of plate edge}

In this section we consider waves generated by a force excitation normal to the edge of a plate. Figure 1 shows the geometry under consideration, a plate of thickness $d$ excited by a distributed force normal to the edge. A good approximation to this situation results when a plate is excited by a conventional piezoelectric transducer using grease coupling (Figure 1, bottom).

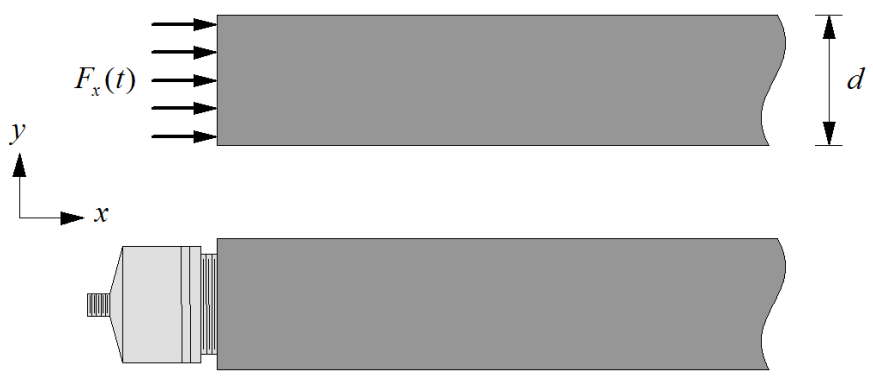

Figure 1. Excitation of the edge of a plate of thickness $d$ : (top) ideal case of excitation by a force normal to the surface; and (bottom) excitation by a grease-coupled transducer.

We first investigate the transition from a low-frequency regime in which only Lamb waves propagate to a high-frequency regime in which only longitudinal waves are generated. We show that the frequency-thickness product need not be very high $(f \cdot d$ need not exceed $15 \mathrm{MHz}-\mathrm{mm})$ in order to create nearly-longitudinal waves that propagate for considerable distances. We also obtain insight into the formation of trailing pulses that are known to form when longitudinal waves propagate in plates.

We first show experimental records of trailing pulses formed when a nearly-longitudinal wave is excited in a plate. We excited a steel plate, $1.9 \mathrm{~cm}$ thick and $30.5 \mathrm{~cm}$ long, by a piezoelectric element of 5A4E material (Piezo Systems, Cambridge, MA), $15 \times 15 \times 1 \mathrm{~mm}$ with a lead backing $3 \mathrm{~mm}$ thick, bonded to the plate with cyanoacrylate adhesive. The transducer is driven by a waveform 


$$
v(t)=\left\{\begin{array}{cl}
v_{0} \sin (2 \pi f t) \cdot\left(\sin \left(\frac{f t}{20 \pi}\right)\right)^{2} & t<\frac{5}{f} \\
0 & t \geq \frac{5}{f}
\end{array}\right.
$$

with a center frequency $f$ of $2.2 \mathrm{MHz}$. Figure 2 shows the signal obtained at a similar transducer on the opposite edge, consisting of a pulse arriving at $t=52.6 \mu \mathrm{s}$ followed by several trailing pulses. The first arrival corresponds to a group velocity of $5.8 \times 10^{5} \mathrm{~cm} / \mathrm{sec}$, which agrees well with the longitudinal velocity $\left(5.9 \times 10^{5} \mathrm{~cm} / \mathrm{sec}\right)$ in steel. This leading pulse is followed by several trailing pulses, equally spaced by approximately $5 \mu \mathrm{s}$. In this instance the leading pulse is the largest, but at different propagation distances or at different center frequencies one of the trailing pulses may be the largest.

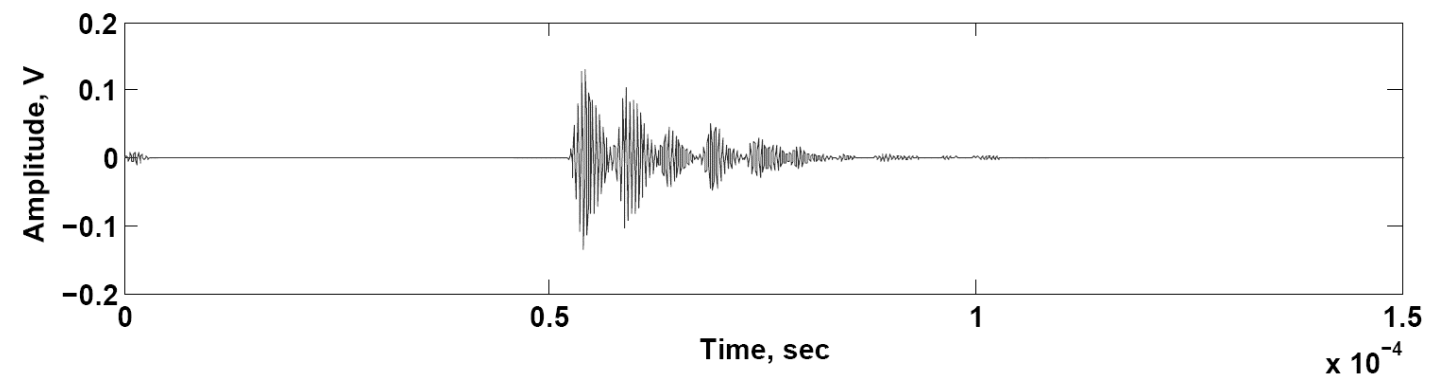

Figure 2. Received signal after a propagation distance of $30.5 \mathrm{~cm}$. The pulse center frequency was 2.2 MHz. The small signal at $t=0$ is due to electrical stray coupling.

We repeated the experiment using a commercial ultrasonic flaw detection system in pitch-catch mode, pulsing a transducer (Krautkramer Gamma series, 0.5-in diameter, 3.5 MHz) with a Krautkramer USPC-2100 system, and those results are shown in Figure 3. The first arrival time in Figure 3 is identical to that in Figure 2, as is the spacing between trailing pulses.

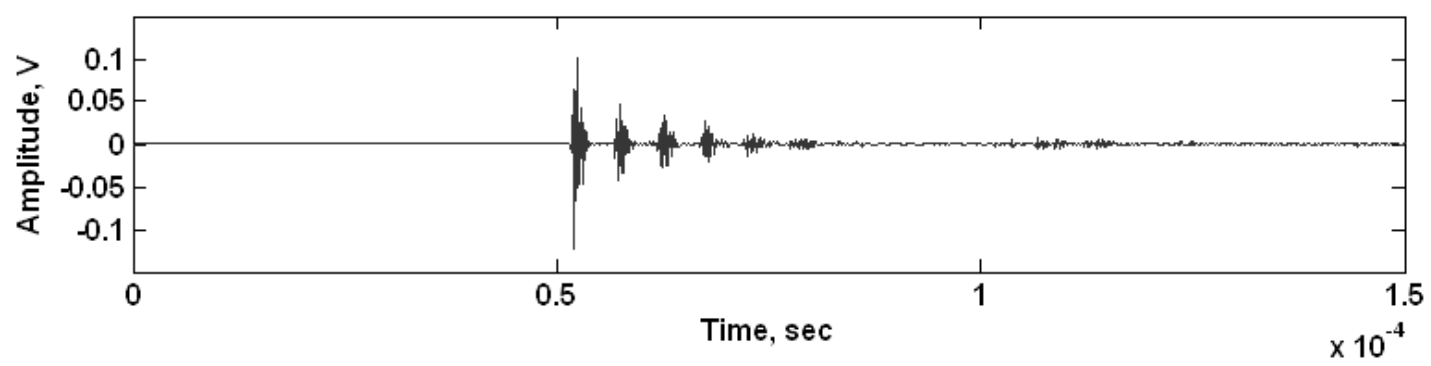

Figure 3. Received signal after a propagation distance of $30.5 \mathrm{~cm}$, excited by Krautrkamer USPC2100 pulser. The small signal at $t=0$ is due to electrical stray coupling.

It is well known that trailing pulses are associated with boundary reflections, having been termed [13] "secondary echoes produced by split-off transverse waves" and explained as shown in Figure 4. Within the bulk wave radiating from the transducer, Figure 4 highlights one longitudinal wave that 
propagates straight down the plate (a) and another longitudinal wave that strikes the plate edge at oblique incidence (b). The latter wave produces a reflected longitudinal wave (c) and a reflected transverse wave (d). Consider now the case of the oblique longitudinal wave (b) approaching the boundary at grazing incidence, such that the reflected longitudinal wave (c) propagates straight down the plate; in that case the reflected transverse wave (d) forms at a critical angle $\theta$, and when it strikes the opposite edge it creates another longitudinal wave (e) propagating straight down the plate but delayed with respect to the original pulse (a), along with a reflected transverse wave (f). The process repeats, resulting in additional trailing longitudinal pulses propagating straight down the plate.

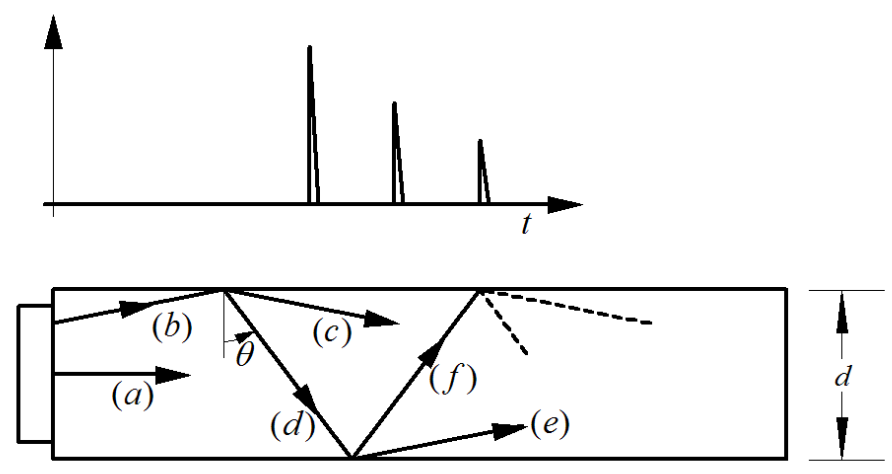

Figure 4 Explanation for the formation of trailing pulses, Krautkramer [8].

In steel, the reflected transverse wave (d) is produced at an angle $\theta \sim 33$ degrees from the normal, and the time separation between successive trailing pulses is calculated as [13]

$$
\Delta t=\frac{d}{c_{t} \cos \theta}-\frac{d \tan \theta}{c_{l}}=4.9 \mu \mathrm{s}
$$

where $c_{t}$ and $c_{l}$ are the transverse and longitudinal wave velocities, respectively. This result is in good agreement with the separation of $5 \mu$ s observed experimentally in Figures 2 and 3.However, this classical picture provides no guidance as to the lowest $f \cdot d$ at which the longitudinal pulse propagates for useful distances, nor does it determine how rapidly the leading pulse decays in amplitude. Indeed, the applicability of the reflection and mode conversion equations is questionable when the plate thickness is only a few wavelengths.

In the following section, we report on our investigations of the generation and propagation of waves in this geometry using finite element simulations. These results provide insight into the generation and propagation of trailing pulses. In addition, these simulations show how a propagating pulse sheds energy by the creation of trailing pulses with an identical shape.

\section{Simulations of wave generation}

Simulations were performed in the plane-strain structural mechanics mode of Comsol 3.2 for a plate 2 $\mathrm{cm}$ thick and $35 \mathrm{~cm}$ long, corresponding to the model shown in Figure 1 with time dependence of force from eq. (1). Material parameters (Young's modulus $E=2 \times 10^{11} \mathrm{~N} / \mathrm{m}^{2}$, Poisson ratio 0.33, density $7850 \mathrm{~kg} / \mathrm{m}^{3}$, mass damping parameter 0.001 and stiffness damping parameter $10^{-9}$ ) were chosen appropriate for steel. A transient (time dependent) analysis was performed using a step size of $1 / 10 f$. The mesh parameters for simulations at $f>600 \mathrm{kHz}$ were chosen to give a maximum element size of $0.2 \mathrm{~cm}$, resulting in approximately 13200 elements, whereas slightly larger element sizes $(0.25$ 
$\mathrm{cm})$ were used at lower frequencies. One simulation at $1 \mathrm{MHz}$ was repeated using a smaller element size of $0.12 \mathrm{~cm}$ in order to verify that the mesh size for other simulations was sufficiently small.

We first consider the effect of pulse center frequency on wave generation. Figure 5 shows the effect of launching a wave with edge excitation at various frequencies by plotting the particle displacement (in the $x$ direction) in the plate at $t=50 \mu \mathrm{s}$. At low frequencies (up to about $450 \mathrm{kHz}$ ) the wavefront velocity is significantly slower than the bulk wave longitudinal velocity $\left(c_{l}=5.9 \times 10^{5} \mathrm{~cm} / \mathrm{sec}\right)$, and particle displacements are similar to those for low-order symmetric Lamb modes. The group velocity for the faster mode, based upon the wavefront position, is estimated as $4.6 \times 10^{5} \mathrm{~cm} / \mathrm{sec}$, and the group velocity for the slower mode is estimated as $3.4 \times 10^{5} \mathrm{~cm} / \mathrm{sec}$. These particular modes can be identified as S1 and S2 modes, respectively, and both of these Lamb wave modes are predicted to be present at $f \cdot d=4 \mathrm{MHz} \cdot \mathrm{mm}$.

At higher $f \cdot d$ products, theory predicts that a greater number of Lamb wave modes will be present with a considerable range of group velocities, and as a result one might expect a long pulse train with complex character. However, the simulation results in Figure 5 for higher frequency excitations show particle velocities that are nearly uniform across the thickness of the plate, traveling with a group velocity essentially equal to the bulk wave longitudinal velocity. This behavior is fully established at $750 \mathrm{kHz}(f \cdot d=15 \mathrm{MHz} \cdot \mathrm{mm})$ and above.

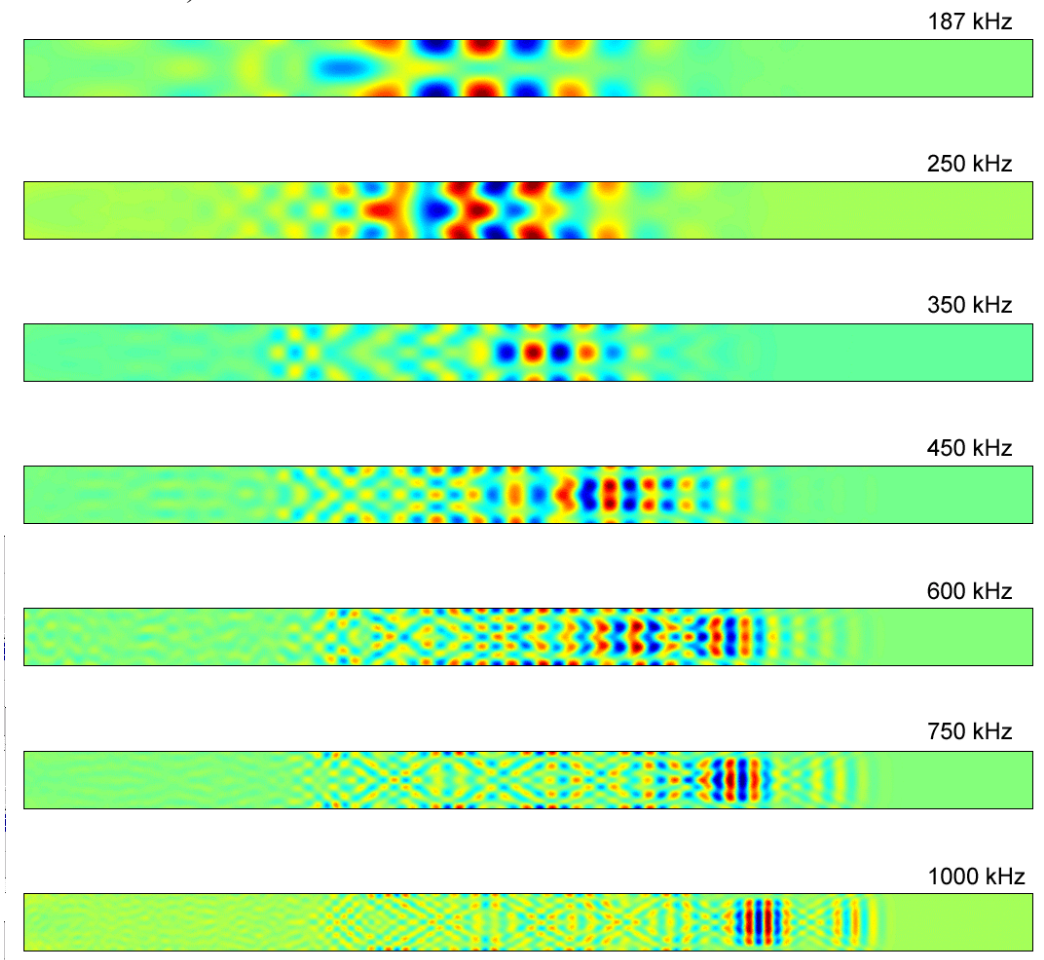

Figure 5. Effect of center frequency on propagating waves for a steel substrate $2 \mathrm{~cm}$ in thickness. The color indicates the particle displacement in the $x$ direction at $t=50 \mu \mathrm{s}$.

In the simulation results at higher $f \cdot d$ shown in Figure 5, the trailing portion of the response has characteristic properties that are quite different from Lamb wave modes. Figure 6 shows snapshots of particle displacement in the $x$ direction at different times for a $1 \mathrm{MHz}$ excitation of a plate $2 \mathrm{~cm}$ thick $(f \cdot d=20 \mathrm{MHz} \cdot \mathrm{mm})$. The simulation shows the formation of a near-planar wavefront within the first 5$10 \mu$ s of propagation. This wavefront propagates at the longitudinal velocity and gradually loses energy while a second trailing pulse forms and gains energy. 


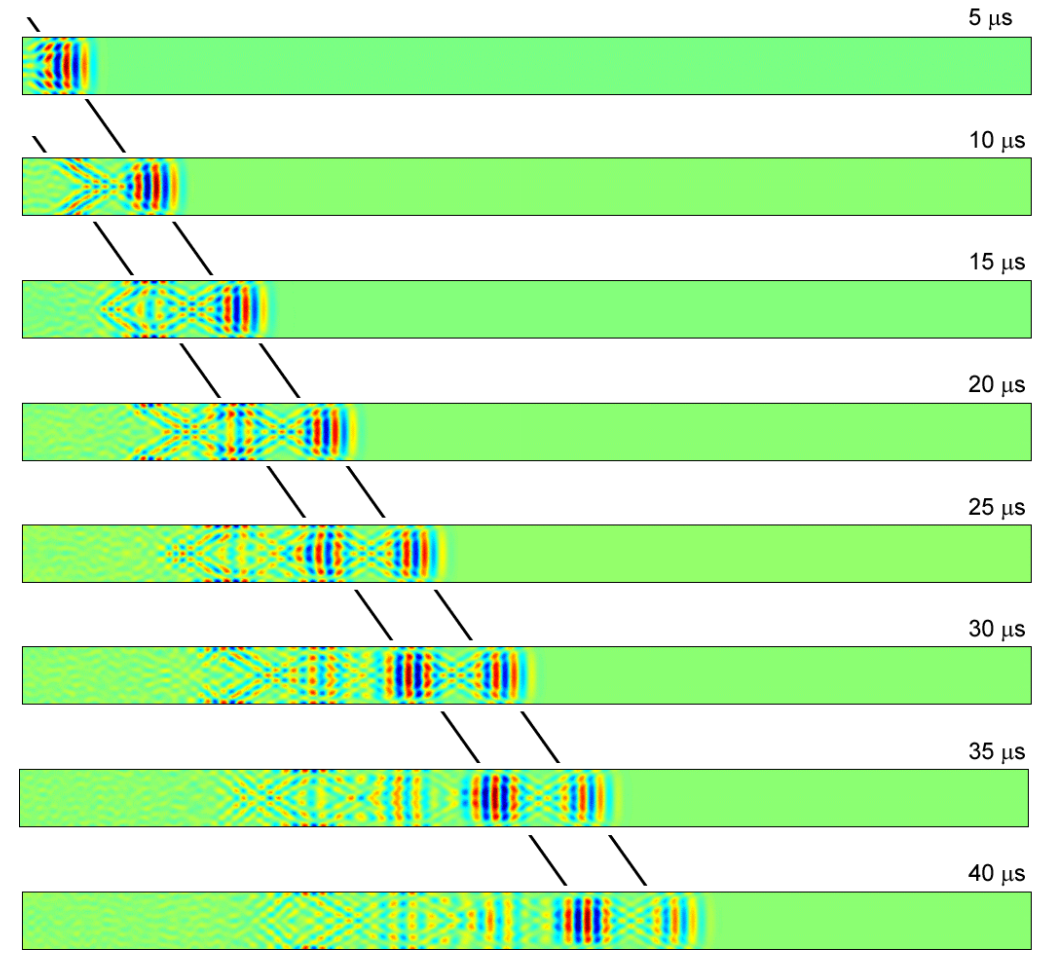

Figure 6. Snapshots showing the $x$ displacement at different times $(2 \mathrm{~cm}$ steel plate, $1 \mathrm{MHz}$ center frequency).

Figure 7 shows in detail the formation of the trailing pulses and their wave structure. The particle displacements at $t=30 \mu \mathrm{s}$ in the $x$ and $y$ direction are plotted at top and bottom, respectively. The particle displacements in the $x$ direction show a nearly-longitudinal pulse that has decayed in amplitude, followed by a second nearly-longitudinal wave packet with largest amplitude, followed by a third wave packet that is forming, followed by a tail resulting from interference of waves reflected from the top and bottom plate surfaces.

More detail of the wave structure can be seen from the plot (Figure 7, bottom) of particle displacements in the $y$ direction. This plot clearly reveals the transverse wave packet described in the simplified model and discussed earlier; the wave crests are marked with dashed lines. The wave packet is propagating in the direction indicated by the arrow, and was generated by the leading wave packet. The propagation direction forms an angle of about 30 degrees with the surface normal, in good agreement with the 33 degree reflection angle expected for a transverse wave produced by a glancingincidence longitudinal wave. We also see that this wave packet has a shorter wavelength than the longitudinal wave packet, consistent with the slower bulk wave transverse velocity. An identical transverse mode wave packet is reflected from the bottom surface. Note that both transverse and longitudinal mode wave packets propagate in the $+x$ direction; however, the longitudinal mode packets move faster and leave the transverse waves behind. The two transverse waves from top and bottom surfaces reflect from the opposite surface and eventually resolve into a trailing longitudinal wave packet. 

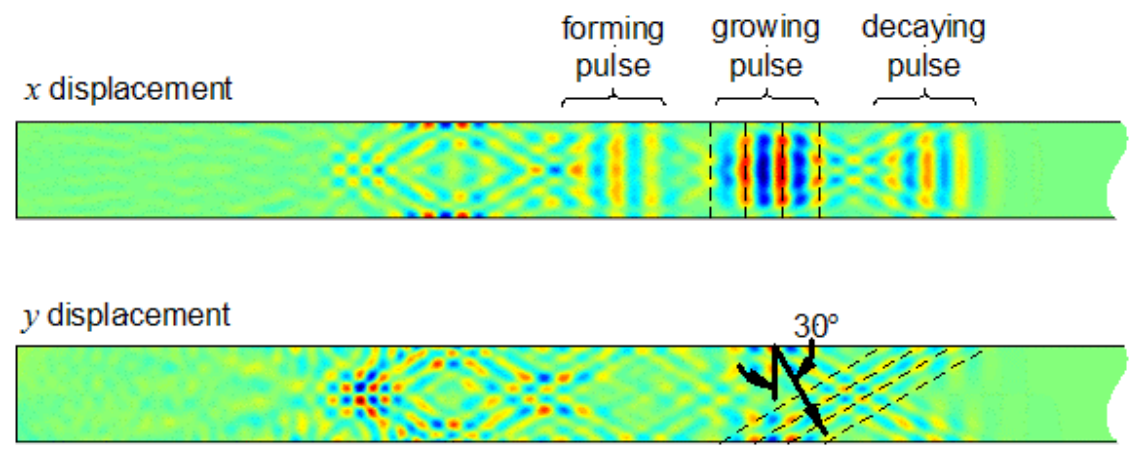

Figure 7. Detail showing the formation of trailing pulses with a center frequency of $1 \mathrm{MHz}$ : (top) $x$ displacement and (bottom) $y$ displacement. Snapshot is $30 \mu$ s after launching and the plate is $2 \mathrm{~cm}$ steel.

The simulations are entirely consistent with the classical explanation of the trailing pulse formation. However, the classical explanation cannot provide information about the rate at which energy is lost to trailing pulses or the lowest $f \cdot d$ product at which this type of wave is formed. In order to study the transfer of energy from the leading pulse to trailing pulses, we evaluated the average $x$ displacement across the entire thickness of the plate at various locations, which is approximately proportional to the voltage that would be produced across a piezoelectric transducer at that location. Typical results obtained for a $2 \mathrm{~cm}$ plate at $1 \mathrm{MHz}$ are presented in Figure 8. The simulations show the decrease in amplitude of the leading pulse and the growth of the second pulse until they have nearly equal amplitude at $x=0.15 \mathrm{~m}$. A similar behavior is seen at other center frequencies. At lower center frequencies the pulses are wider and begin to overlap, while at higher frequencies at least five distinct trailing pulses can be seen after a propagation distance of $0.30 \mathrm{~m}$. 


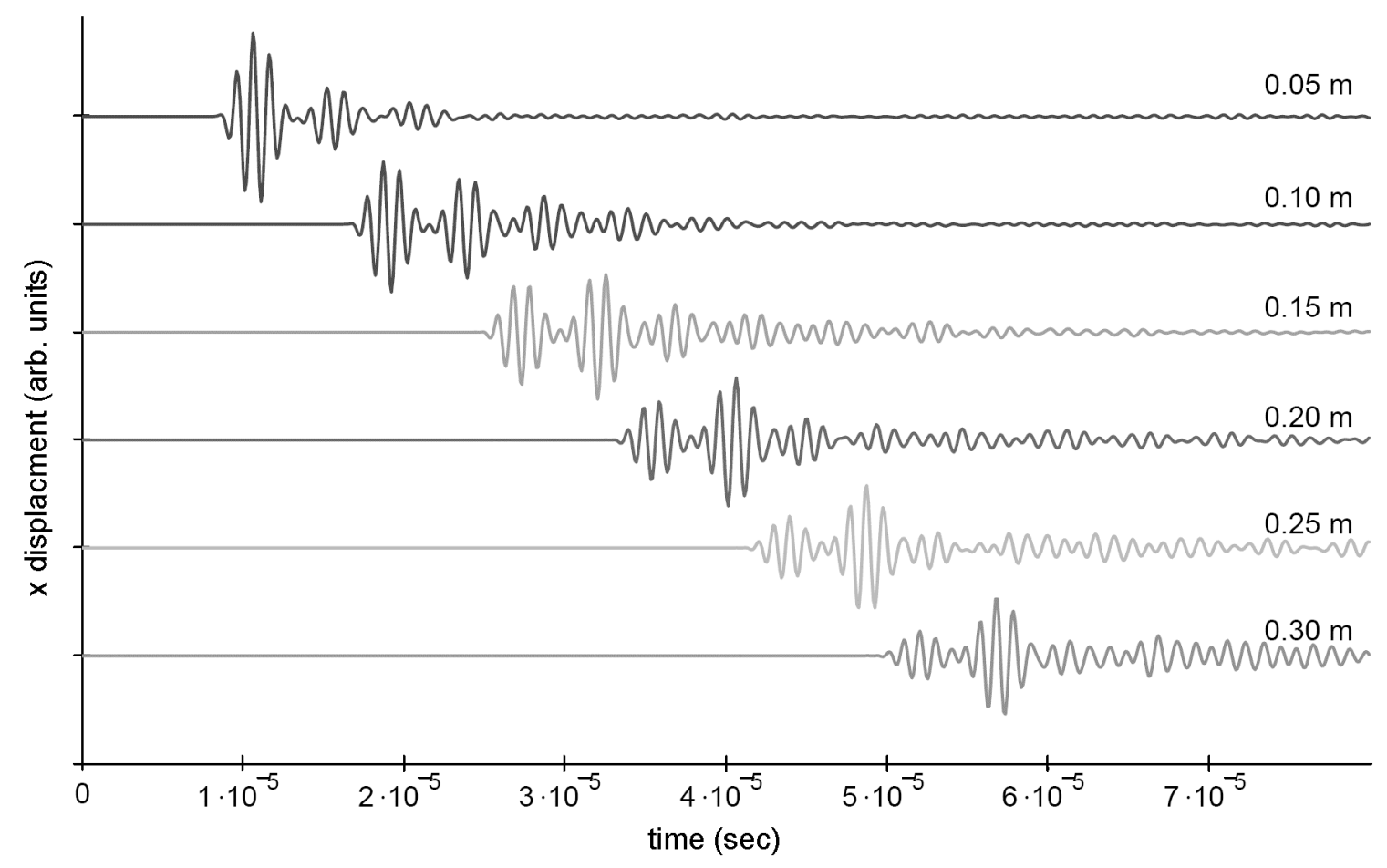

Figure 8. Average $x$ displacement across the plate for a $1 \mathrm{MHz}$ center frequency pulse as a function of time.

These simulations have been performed at several center frequencies and there is a clear trend in the rate at which energy is transferred from the leading to the trailing pulses. This can be seen by comparing the propagation distance at which the first and second pulses become equal in magnitude (Table I). This transfer of energy becomes less rapid as the frequency increases. This is consistent with Figure 2, where the first pulse is largest in amplitude for a propagation distance of $30.3 \mathrm{~cm}$ with an $f \cdot d$ product of $42 \mathrm{MHz} \cdot \mathrm{mm}$.

Table I. Propagation distance at which the leading and first trailing pulses are equal in amplitude.

\begin{tabular}{ccc} 
frequency $(\mathrm{MHz})$ & $f \cdot d(\mathrm{~mm} \cdot \mathrm{MHz})$ & distance $(\mathrm{m})$ \\
\hline .075 & 15 & $<0.1$ \\
1.00 & 20 & $<0.15$ \\
1.25 & 25 & 0.2 \\
1.5 & 30 & 0.3 \\
\hline
\end{tabular}

\section{Scattering from cracks}

Finally we consider scattering of nearly-longitudinal waves from a crack. It is possible that scattering from a crack would result in mode conversion to multiple Lamb wave modes, which would make detection of cracks substantially more difficult. Therefore, we undertook simulation and experimental studies to determine whether such unwanted mode conversion occurs. 
In order to observe such mode conversion, were it to occur, simulations were performed to include the effect of scattering from a part-thickness slot $1 \mathrm{~mm}$ wide and with varying depth. The simulations were performed with a center frequency of $1 \mathrm{MHz}$ for a plate $2 \mathrm{~cm}$ thick and $0.4 \mathrm{~m}$ long with a crack located $0.2 \mathrm{~m}$ from the left edge at which the excitation was applied. Figure 9 shows a nearlylongitudinal wave approaching the crack (top, $t=30 \mu \mathrm{s}$ ) and the transmitted and reflected waves (bottom, $t=50 \mu \mathrm{s}$ ).
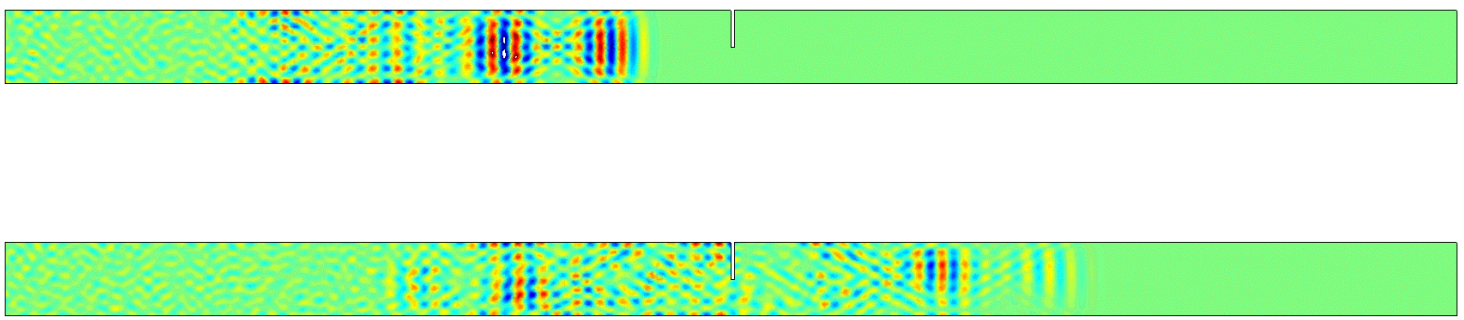

Figure 9. Interaction of a pulse train, center frequency $1 \mathrm{MHz}$, with a half-thickness crack $1 \mathrm{~mm}$ wide: (top) $t=30 \mu \mathrm{s}$; (bottom) $t=50 \mu \mathrm{s}$ ).

Figure 10, top and bottom, shows the average $x$ displacement as a function of time at two locations, $x=0.10 \mathrm{~m}$ and $x=0.30 \mathrm{~m}$, respectively, symmetrically positioned with respect to the crack. Figure 10 (top) shows the incident wave approaching the first location $(x=0.10 \mathrm{~m})$ at $t \sim 17 \mu \mathrm{s}$, and a wave reflected from the crack approaching that same location on its return path at $t \sim 51 \mu$ s. Figure 10 (bottom) shows the transmitted wave, after having passed the scatterer, approaching the second location $(x=0.30 \mathrm{~m})$ at $t \sim 51 \mu$ s with nearly the same amplitude and shape as the reflected wave returning to the first location. Examining the wave arriving at the second location $(x=0.30 \mathrm{~m})$, and its counterpart reflected wave returning to the first location, we note that the second pulse is larger than the leading pulse, consistent with the simulations for a total travel distance of $0.30 \mathrm{~m}$ without a crack as shown in Fig. 9. The expected trend with crack depth is observed, with the ratio of reflected wave amplitude increasing monotonically with increasing crack depth (not shown).

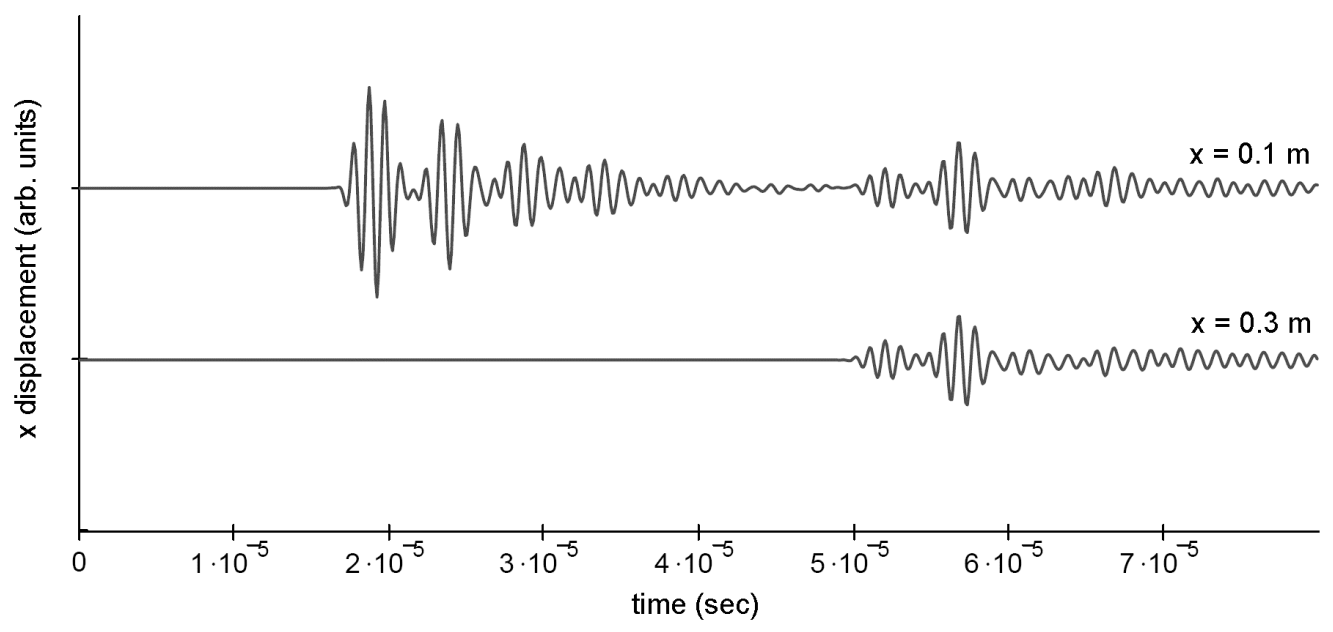

Figure 10. Average $x$ velocity as a function of time at two locations, slot located at $x=0.2 \mathrm{~m}$. 
Experiments confirm that nearly-longitudinal waves retain their most-important characteristics (velocity, trailing pulse train, and pulse shape) when reflected from a crack. Figure 11 shows the response recorded at the exciting transducer for a steel plate $1.9 \mathrm{~cm}$ thick and $0.305 \mathrm{~m}$ long with a 12.6 $\mathrm{cm}$ wide slot $5.7 \mathrm{~mm}$ in depth located midway along the length. A nearly-longitudinal wave with trailing pulses, reflected from the crack, approaches the transducer starting at $t \sim 50 \mu \mathrm{s}$. Another nearly-longitudinal wave with trailing pulses approaches the transducer starting at $t \sim 100 \mu \mathrm{s}$; it consists of the original wave reflecting from the opposite edge and twice scattered, together with the wave first reflected from the crack being reflected from the transducer edge and then a second time from the crack. The two wave trains are quite similar and show the same characteristics of nearlylongitudinal waves. From the simulations and experiments we make the important observation that nearly-longitudinal waves retain their velocity and pulse shape when scattered.

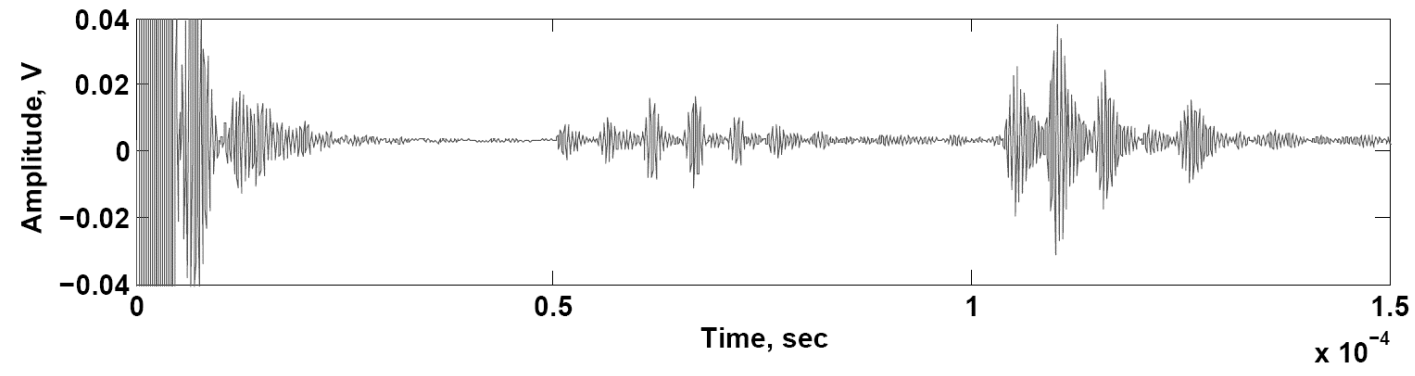

Figure 11. Experimentally observed scattering of a pulse train from a slot. The pulse train beginning at $50 \mu \mathrm{s}$ is due to scattering from the slot, and the pulse train beginning shortly after $100 \mu \mathrm{s}$ is due to reflection from the opposite edge.

\section{Summary}

We have examined the generation of waves in plates by force excitation normal to the edge as a function of pulse center frequency. Both simulations and experiment show that multiple Lamb modes are created at low $f \cdot d$ products, while for $f \cdot d>15 \mathrm{MHz} \cdot \mathrm{mm}$ a nearly longitudinal pulse is generated which is followed by several trailing pulses. During propagation, energy is transferred from the leading pulse to trailing pulses. Our simulations show that the rate of transfer of energy from leading to trailing pulses decreases with increasing center frequency. Both experimental results and simulations show that the pulse train retains its integrity over distances of at least one meter. We have also studied the interaction of a pulse train with a slot, simulating scattering from a partial-thickness crack. Both transmitted and reflected pulse trains are observed and there is no sign of mode conversion to Lamb waves.

We suggest that these nearly-longitudinal waves are attractive as an alternative to Lamb waves for the detection of defects in plate-like structures. An important advantage of the nearly-longitudinal modes is the shorter wavelength which will result in enhanced scattering from small defects. Reflection of a pulse train rather than a single pulse will complicate the interpretation of data. We suggest that this complication will be acceptable in order to take advantage of higher scattering amplitude.

\section{Acknowledgements}

The authors gratefully acknowledge support from Bombardier Total Transit Systems and the Pennsylvania Infrastructure Technology Alliance, and from the National Science Foundation under grant CMS-0329880. Any opinions, findings, and conclusions or recommendations expressed in this material are those of the authors and do not necessarily reflect the views of the National Science Foundation. 


\section{References}

1. Moore M, Phares B, Graybeal B, Rolander D, and Washer G, 2001 Reliability of Visual Inspection for Highway Bridges, Volume 1: Final Report and, Volume II: Appendices, FHWA-RD-01-020 and $\mathbf{- 0 2 1}$

2. Alleyne D N and Cawley P 1992 NDT \& E Int. 25, 11-22

3. Ihn J-B and Chang F-K 2004 Smart Mater. Struct. 13, 609-20

4. Pierce S G, Culshaw B, Manson G, Worden K, and Staszewski W J 2000 Proc. SPIE - The International Society for Optical Engineering 3986 93-103

5. Rose J L, Pelts S, and Quarry M 1998 Ultrasonics 36, 163-8

6. Zhu W and Rose J L 1999 IEEE Trans. Ultrasonics, Ferroelectrics, and Frequency Control 46, 654-64

7. Giurgiutiu V 2003 Proc. SPIE- Smart Structures and Materials 2003: Smart Structures and Integrated Systems 5056 111-22

8. Fromme P, Masserey B, and Sayir M B 2003 Rev. Prog. Quantitative Nondestructive Evaluation 22, 189-96

9. Lee B C and Staszewski W J 2007 Smart Mater. Struct. 16 249-59

10. Lee B C and Staszewski W J 2007 Smart Mater. Struct. 16 260-74

11. Lee B C and Staszewski W J 2007 Smart Mater. Struct. 16 399-408

12 Greve D W, Oppenheim I J, Wu, W and Zheng P 2007 Proc. Sensors and Smart Structures Technologies for Civil, Mechanical, and Aerospace Systems $\mathbf{6 5 2 9}$

13. Krautkramer J and Krautkramer H 1990 Ultrasonic Testing of Materials 4th fully revised edition, Springer-Verlag 283-6 